\title{
P300/CBP Bromodomain Inhibitor CCS1477
}

National Cancer Institute

\section{Source}

National Cancer Institute. p300/CBP Bromodomain Inhibitor CCS1477. NCI Thesaurus.

Code C160195.

An orally bioavailable, small molecule inhibitor of the highly conserved bromodomains of the histone acetyltransferase (HAT) paralogs, p300 (E1A-associated protein p300; p300 HAT) and CREB binding protein (CBP), with potential antineoplastic activity. Upon oral administration, p300/CBP bromodomain inhibitor CCS1477 selectively and reversibly binds to the bromodomains of p300 and CBP. This disrupts the acetylation of histones and other proteins and prevents the co-activation of key transcription factors that contribute to tumor progression including the androgen receptor (AR), androgen receptor splice variants (AR-SV), hypoxia-inducible factor 1-alpha (HIF-1-alpha) and Myc proto-oncogene protein (c-Myc). The HAT paralogs p300 and CBP are key transcriptional co-activators that are essential for a multitude of cellular processes and are implicated in the progression and therapeutic resistance of certain cancers. 